\title{
Images
}

\section{A typical case of divided nevus of the eyelid}

\section{Sun Zhi-Yong ${ }^{1,2}$, Zan Tao'2, Sheng Guo-Xiong'2, Li Qing-Feng ${ }^{2}$}

${ }^{1}$ Department of Plastic Surgery, The First Affiliated Hospital of Guangxi Medical University, Nanning 530021, China

${ }^{2}$ Department of Plastic and Reconstructive Surgery, Shanghai $9^{\text {th }}$ Hospital, Shanghai Jiaotong University, Shanghai 200011, China

Address for correspondence: Dr. Sun Zhi-Yong, Department of Plastic Surgery, The First Affiliated Hospital of Guangxi Medical University, Nanning 530021, China. E-mail: drzhiyongsun@yahoo.com

seven-year-old boy presented with a diagnosed congenital divided nevus of the eyelid, which was a continuous nevus that affects the upper and lower eyelid [Figures A and B]. Divided nevus of the eyelid is a rare pathologic entity and develops during foetal growth, when the two eyelids are still fused together. Its surgical treatment is mostly based on full thickness skin grafts
[Figure D]. This case of divided nevus of the eyelid was typical and worth taking a photograph, unexpectedly, the intelligent eye reflecting the doctor perfectly [Figures. B and $\mathrm{C}$.

Editorial Note: This is just a set of photos but they say more than a thousand words!

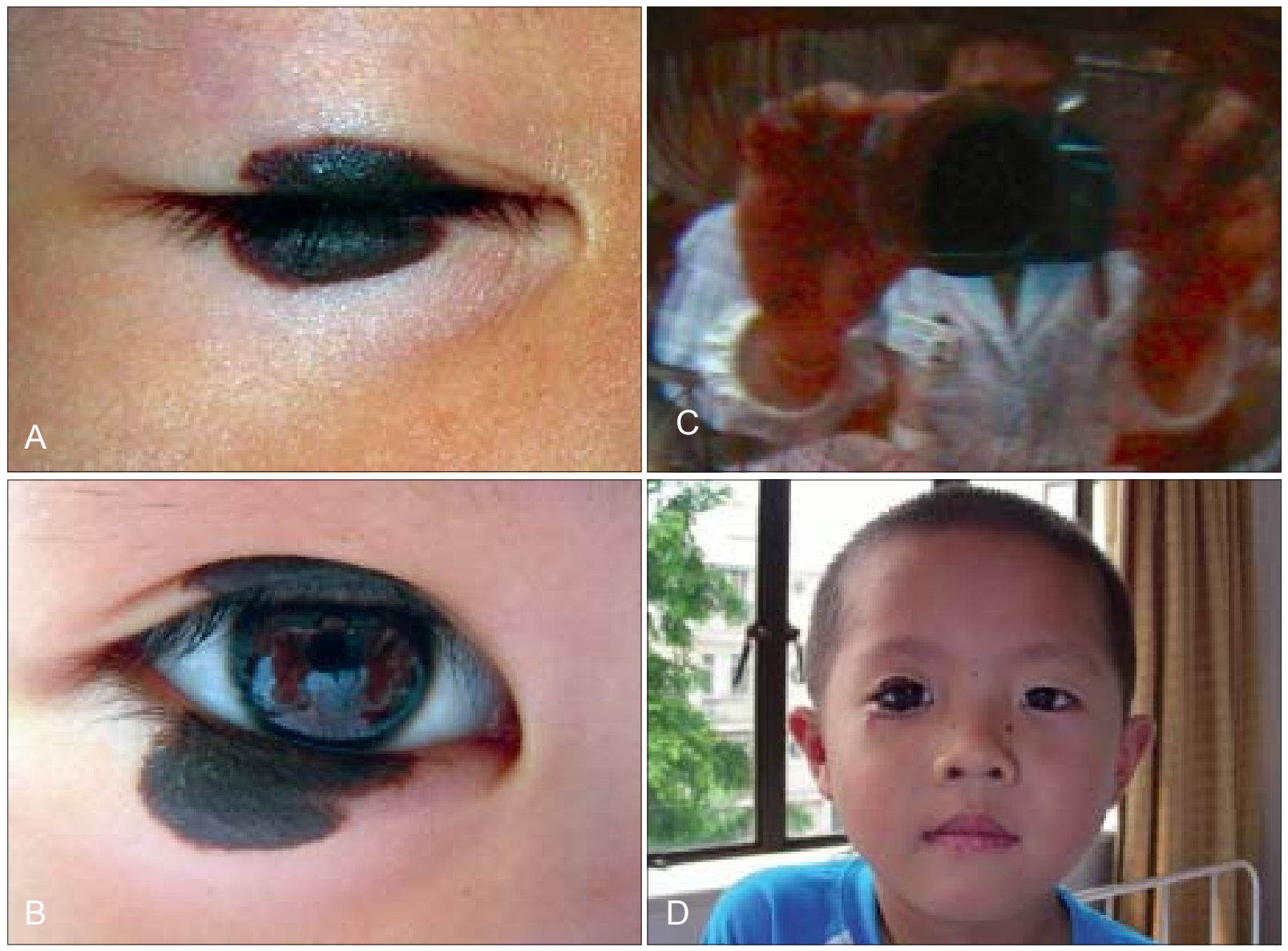

Figure: (A-D) A 7-year-old boy presented with a diagnosed congenital divided nevus of the eyelid, which was a continuous nevus that affects the upper and lower eyelid (Panels A and B). Divided nevus of the eyelid is a rare pathologic entity and develops during foetal growth, when the two eyelids are still fused together. Its surgical treatment is mostly based on full thickness skin grafts (Panels $D$ ). This case of divided nevus of the eyelid was typical and worth taking a photograph, unexpectedly, the intelligent eye reflecting the doctor perfectly (Panels B and C) 